SLAC-PUB-6506

May 1994

hep-th/9407039

$\mathrm{T}$

\title{
Towards Quantum Cosmology without Singularities ${ }^{\dagger}$
}

\author{
Klaus Behrndt ${ }^{\ddagger}$ and Thomas T. Burwick ${ }^{\S}$ \\ Stanford Linear Accelerator Center \\ Stanford University, Stanford, California 94309
}

\begin{abstract}
In this paper we investigate the vanishing of cosmological singularities by quantization. Starting from a 5d Kaluza-Klein approach we quantize, as a first step, the non-spherical metric part and the dilaton field. These fields which are classically singular become smooth after quantization. In addition, we argue that the incorporation of non perturbative quantum corrections form a dilaton potential. Technically, the procedure corresponds to the quantization of $2 \mathrm{~d}$ dilaton gravity and we discuss several models. From the $4 \mathrm{~d}$ point of view this procedure is a semiclassical approach where only the dilaton and moduli matter fields are quantized.
\end{abstract}

We consider a cosmological string solution which has classical singularities (big bang). Near these singularities the theory factorizes in a smooth spherical part and a singular $2 \mathrm{~d}$ part. This singular part is the well-known dilaton gravity (see e.g. [1]-[4]) and as a first step we are going to quantize this part with the result that all singularities disappear. This procedure is also known as s-wave reduction and was so far used for $4 \mathrm{~d} \mathrm{BH}$ physics.

1. Classical theory. Our $4 \mathrm{~d}$ classical model is given by

$$
S=\int d^{4} x \sqrt{\tilde{G}} e^{-2 \phi}\left(R+4(\partial \phi)^{2}-\left(\frac{\partial \rho}{\rho}\right)^{2}-\frac{1}{12} H^{2}\right)
$$

where $\phi$ is a dilaton field, $H_{\mu \nu \lambda}=\partial_{[\mu} B_{\nu \lambda]}$ is the torsion corresponding to the antisymmetric tensor field $B_{\mu \nu}$ and $\rho$ is a modulus field. A cosmological solution to this model is given

\footnotetext{
${ }^{\dagger}$ Work supported by the Department of Energy, contract DE-AC03-76SF00515.

${ }^{\ddagger}$ e-mail: behrndt@jupiter.slac.stanford.edu

$\S$ e-mail: burwick@slacvm.slac.stanford.edu
} 

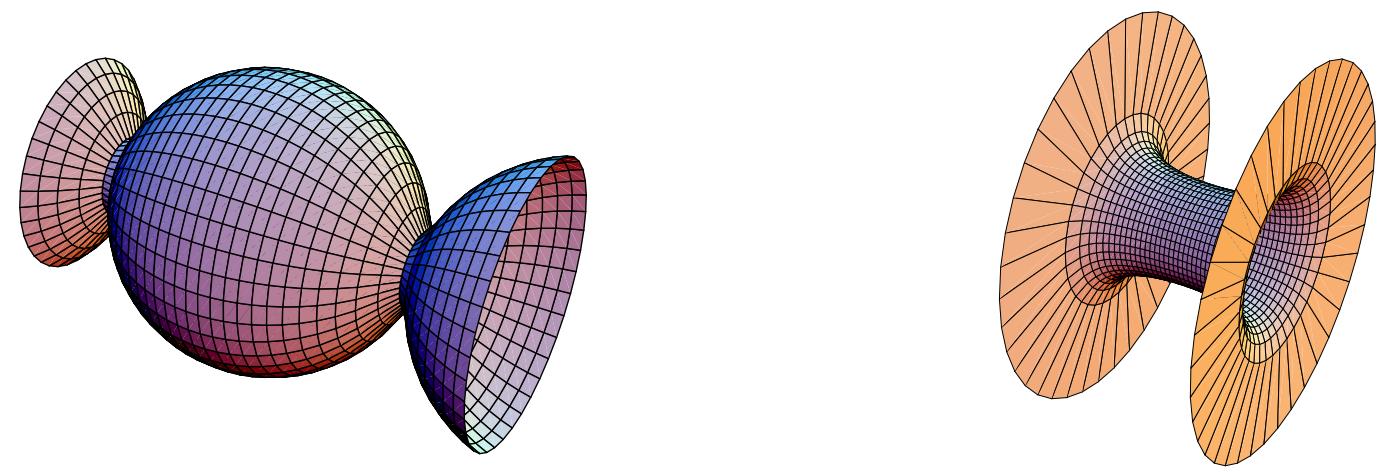

Figure 1: In (a) we have plotted the closed oscillating solution for $k=1$ and (b) is the wormhole solution for $k=-1$.

by [5]

$$
\begin{aligned}
& d s^{2}=-\frac{d t^{2}}{\left(-k+\left(\frac{t_{+}}{t}\right)^{2}\right)\left(1-\left(\frac{t_{-}}{t}\right)^{2}\right)}+t^{2} d \Omega_{k}^{2} \quad, \quad \rho^{2}=\frac{-k+\left(\frac{t_{+}}{t}\right)^{2}}{1-\left(\frac{t_{-}}{t}\right)^{2}} \\
& H=2 t_{+} t_{-}\left(\frac{\sin \sqrt{k} \chi}{\sqrt{k}}\right)^{2} \sin \theta d \chi \wedge d \theta \wedge d \varphi \quad, \quad e^{-2 \phi} \sim \sqrt{\left(1-\left(\frac{t_{-}}{t}\right)^{2}\right)\left(-k+\left(\frac{t_{+}}{t}\right)^{2}\right)} .
\end{aligned}
$$

After a time reparameterization one obtains the standard Friedmann-Robertson-Walker (FRW) metric with $d \Omega_{k}^{2}$ as $3 \mathrm{~d}$ volume form corresponding to the spatial curvature $k$ (= $0,-1,+1)$. The parameter $t_{-}$is the minimal extension and for $k=1$ the parameter $t_{+}$ denotes the maximal extension of the universe. This is obvious after transforming the solution to the conformal time

$$
t^{2}=t_{-}^{2}+\left(t_{+}^{2}-k t_{-}^{2}\right)\left(\frac{\sin \sqrt{k} \eta}{\sqrt{k}}\right)^{2}
$$

for which the metric is

$$
d s^{2}=\left\{t_{-}^{2}+\left(t_{+}^{2}-k t_{-}^{2}\right)\left(\frac{\sin \sqrt{k} \eta}{\sqrt{k}}\right)^{2}\right\}\left[-d \eta^{2}+d \Omega_{k}^{2}\right] .
$$

Unfortunately, in [5] no analytic results for the world radius $a(\tau)$ in the standard parameterization of the FRW metric $d s^{2}=-d \tau^{2}+a(\tau)^{2} d \Omega_{k}^{2}$ could be found. We have plotted numerical results in figure 1. Figure 1 a shows the oscillating solution for $k=1$ and $1 \mathrm{~b}$ the wormhole solution for $k=-1$. For $k=0$ the solution has again the geometry of figure $1 \mathrm{~b}$ but with the difference that there are no asymptotic flat regions as for $k=-1$. 
Remarkably, the scalar fields $\rho$ and $\phi$ have divergencies although the metric behaves completely smooth for all times. To understand this phenomena one has to go back to the 5 dimensional $(5 \mathrm{~d})$ origin. In the sense of a Kaluza-Klein approach solution (2) can be obtained by dimensional reduction of a $5 \mathrm{~d}$ theory. Then, the modulus field $\rho$ corresponds to a time dependent compactification radius of the fifth coordinate. The corresponding action is given by the effective string action

$$
S^{(5)}=\int d^{5} x \sqrt{G} e^{-2 \psi}\left(R+4(\partial \psi)^{2}-\frac{1}{12} H^{2}\right)
$$

and the 5 dimensional solution can be written as

$$
\begin{aligned}
& d s^{2}=\left(\frac{\sqrt{k}}{\tan \sqrt{k} \eta}\right)^{2} d w^{2}+\left\{t_{-}^{2}+\left(t_{+}^{2}-k t_{-}^{2}\right)\left(\frac{\sin \sqrt{k} \eta}{\sqrt{k}}\right)^{2}\right\}\left[-d \eta^{2}+d \Omega_{k}^{2}\right] \\
& e^{2\left(\psi-\psi_{0}\right)}=1+\frac{t_{-}^{2}}{\left(t_{+}^{2}-k t_{-}^{2}\right)\left(\frac{\sin \sqrt{k} \eta}{\sqrt{k}}\right)^{2}} .
\end{aligned}
$$

The 5 d dilaton is related to $\phi$ by $2 \psi=2 \phi+\log \chi$ and $\rho=G_{55}$. For $k=1$ and after switching the signature of the metric $\left(d w^{2} \rightarrow-d w^{2}\right.$ and $\left.d \eta^{2} \rightarrow-d \eta^{2}\right)$ this $5 \mathrm{~d}$ solution is just the $5 \mathrm{~d} \mathrm{BH}$ solution (in the conformal time $\eta$ ) discussed by Horowitz and Strominger in [6]. Here, $t_{ \pm}$define the two horizons of the theory and our cosmological solution lives between these horizons.

2. S-wave reduction. We are particularly interested in the fate of the singularities if one starts to quantize the theory. Therefore, it is reasonable to restrict ourselves to the region near the singularities $(\sin \sqrt{k} \eta \simeq 0$ ). In this region one can assume that quantum corrections become important. Furthermore, as one can see from (6) in this limit the 5d solution decouples in a $3 \mathrm{~d}$ spherical part $\left(\sim d \Omega_{k}^{2}\right)$ and a $2 \mathrm{~d}(w, \eta)$ part which is the known dual $2 \mathrm{~d} \mathrm{BH} \mathrm{[7].} \mathrm{In} \mathrm{the} \mathrm{figure} \mathrm{it} \mathrm{is} \mathrm{just} \mathrm{the} \mathrm{region} \mathrm{of} \mathrm{minimal} \mathrm{extension,} \mathrm{e.g.} \mathrm{inside} \mathrm{the}$ wormhole of fig. $1 \mathrm{~b}$.

Before we can start to quantize the $2 \mathrm{~d}$ part we have to reduce the $5 \mathrm{~d}$ action (5) down to a $2 \mathrm{~d}$ theory. Motivated is this procedure by the assumption that the quantum corrections respect the spherical symmetry. Generally, this is not the case but sufficient for a first approximation. In $\mathrm{BH}$ physics this procedure is also known as $\mathrm{s}^{-}$wave reduction. For the 5d metric we make the ansatz

$$
d s^{2}=g_{a b}^{(2)} d z^{a} d z^{b}+e^{2 \chi} d \Omega_{k}^{2}
$$

where $g_{a b}^{(2)}$ is the $2 \mathrm{~d}$ metric part. In what follows we quantize only $g_{a b}^{(2)}$ and the dilaton $\psi$ (resp. $\phi$, see below). The remaining fields are assumed to be classical backgrounds given by (2) or (6). After integrating out the angular degrees of freedom and using the $H$ field from (2) we obtain for (5)

$$
S^{(2)}=\int d^{2} z \sqrt{g} e^{-2 \phi}\left(R^{(2)}+4(\partial \phi)^{2}-3(\partial \chi)^{2}+V(\chi)\right)
$$


with $\phi=\psi+\frac{3}{2} \chi$ and $V(\chi)=6 k e^{-2 \chi}-2 t_{+}^{2} t_{-}^{2} e^{-6 \chi}$. Near the singularity $(\eta \simeq 0)$ the background field $\chi$ is smooth, $\left.\frac{\partial}{\partial \eta} \chi\right|_{0}=0$ (see (6) and (17)) and up to the second order in $\eta$ we can approximate the $\chi$ terms by a constant

$$
S^{(2)}=\int d^{2} z \sqrt{g} e^{-2 \phi}\left(R^{(2)}+4(\partial \phi)^{2}+\lambda\right)
$$

with $\lambda=\frac{2}{t_{-}^{2}}\left(3 k-\left(\frac{t_{+}}{t_{-}}\right)^{2}\right)$. A classical solution in conformal coordinates is given by [7]

$$
d s^{2}=e^{2 \sigma} d z^{+} d z^{-} \quad, \quad e^{-2 \phi} \sim e^{-2 \sigma}=u-\lambda z^{+} z^{-}
$$

where $u$ is constant. This solution can be transformed to the $2 \mathrm{~d}(w, \eta)$ part of (6) where $\eta \simeq 0$ corresponds to $u \simeq \lambda z^{+} z^{-}$.

3. Quantization. The quantization of (9) has been studied in various papers [1]-[四, 8, 9]. Each of these models will be discussed, but before we do so in detail let us come back to the classical solution once more. The fact that (6) or (2) are independent of tha fifth coordinate leads to the dual solution

$$
\begin{aligned}
& d s^{2}=\left(\frac{\tan \sqrt{k} \eta}{\sqrt{k}}\right)^{2} d w^{2}+\left\{t_{-}^{2}+\left(t_{+}^{2}-k t_{-}^{2}\right)\left(\frac{\sin \sqrt{k} \eta}{\sqrt{k}}\right)^{2}\right\}\left[-d \eta^{2}+d \Omega_{k}^{2}\right] \\
& e^{2\left(\psi-\psi_{0}\right)}=\left(\frac{\tan \sqrt{k} \eta}{\sqrt{k}}\right)^{2}+\frac{t_{-}^{2}}{\left(t_{+}^{2}-k t_{-}^{2}\right)(\cos \sqrt{k} \eta)^{2}}
\end{aligned}
$$

Both solutions have a significant difference. Singular points of (6) are regular in (11) and vice versa. Furthermore, in the region that we are interested in $(\sin \sqrt{k} \eta \simeq 0)$ the solution (6) is in the strong coupling region $\left(e^{2 \psi} \rightarrow \infty\right)$ whereas the dual solution (11) is in the weak coupling region $\left(e^{2 \psi}<<1\right.$; if we assume that $t_{+}>>t_{-}$which is reasonable, since $t_{ \pm}$corresponds to the maximal/minimal extension of the universe) Again the $2 \mathrm{~d}$ part decouples and can be transformed into (10) where $\eta \simeq 0$ corresponds to $z^{+} z^{-} \simeq 0$.

When quantizating this theory we are especially interested in what happens in the strong coupling region, i.e. the fate of the singularity in (6). As a consistency condition of this procedure we have to ensure that in the classical limit (weak coupling region) we get back our classical result (11) which is non singular for $\eta \simeq 0$. We are following the procedure of de Alwis [8] and later on we discuss the modification concerning the other models. After choosing the conformal gauge

$$
g_{a b}=e^{2 \sigma} \hat{g}_{a b}
$$

we can rewrite (9) as a general $2 \mathrm{~d} \sigma$ model

$$
S=-\int d^{2} z \sqrt{\hat{g}}\left[\hat{g}^{a b} \partial_{a} X^{\mu} \partial_{b} X^{\nu} G_{\mu \nu}(X)+\hat{R} \Phi(X)+T(X)\right]
$$

with: $X^{\mu}=\{\phi, \sigma\}$. Thus, the quantization of the dilaton gravity is reduced to the quantization of a $2 \mathrm{~d} \sigma$ model with the target space spanned by $\phi$ and $\sigma$. This model, however, is 
well defined only if the background fields $G_{\mu \nu}, \Phi$ and $T$ define a $2 \mathrm{~d}$ conformal field theory. This symmetry is a consequence of the fact, that the original theory depends only on $g$ and not on $\hat{g}$, and thus, has to respect the symmetry: $\hat{g} \rightarrow e^{2 \rho} \hat{g}$ and $\sigma \rightarrow \sigma-\rho$ (see (12)). We transform the theory to an exact model and define the quantum theory by this (exact) conformal field theory (see e.g. [8, 9]). Following this approach we first note that the target space metric $G_{\mu \nu}$ has the general structure

$$
d S^{2}=-4 e^{-2 \phi}[1+h(\phi)] d \phi^{2}+4 e^{-2 \phi}[1+\bar{h}(\phi)] d \sigma d \phi+\kappa d \sigma^{2}
$$

where $h$ and $\hat{h}$ are model dependent functions of $\phi$ or $X^{1}$. For $h=\bar{h}=0$ we have the CGHS model [1]; for $2 h=\bar{h}=-e^{2 \phi}$ the model from Strominger [3]; $h=0$ and $\bar{h}=-\frac{\kappa}{4} e^{2 \phi}$ describes the RST model [4]. The parameter $\kappa=\frac{24-N}{6}$ originates from the definition of the functional integration measure and $N$ corresponds to additional conformal matter. As next step we introduce new target space coordinates

$$
\begin{aligned}
& x=\frac{2}{\sqrt{\kappa}} \int d \phi e^{-2 \phi} \sqrt{(1+\bar{h})^{2}+\kappa e^{2 \phi}(1+h)} \\
& y=-\sqrt{\kappa}\left(\sigma-\frac{1}{\kappa} e^{-2 \phi}+\frac{2}{\kappa} \int d \phi e^{-2 \phi} \bar{h}\right)
\end{aligned}
$$

and obtain a flat metric

$$
d S^{2}=-d x^{2}+d y^{2} .
$$

(for negative $\kappa$ we have to perform a Wick rotation in $x$ and $y$ ). For this flat metric it is easy to find the dilaton $\Phi$ and tachyon $T$ that define a conformal field theory. The general solution of the corresponding $\beta$ equations is

$$
\begin{array}{lll}
\Phi(x)=a x+b y & \text { with } & a^{2}-b^{2}=-\kappa \\
T(x) \sim e^{\alpha x+\beta y} & \text { with } & \frac{1}{2}\left(\alpha^{2}-\beta^{2}\right)-a \alpha+b \beta-2=0 .
\end{array}
$$

The demand to get the classical model (9) in the weak coupling limit yields a further restriction to $\Phi$ and $T$. Following the suggestion of de Alwis we set: $a=0$ and $\alpha=-\beta=$ $-\frac{2}{\sqrt{\kappa}}$ and get the known Liouville theory ( $y$ as Liouville field) that couples to the matter field $x$

$$
S=-\int d^{2} z \sqrt{\hat{g}}\left[-(\partial x)^{2}+(\partial y)^{2}+\sqrt{\kappa} \hat{R} y+\lambda e^{-\frac{2}{\sqrt{\kappa}}(x-y)}\right] .
$$

This describes a well defined $2 \mathrm{~d}$ gravity theory on the classical as well as on the quantum level. The strategy is to define the quantum theory in terms of this action and to regard (9) as the classical limit.

As second step we have to find solutions of the equations of motion for $x$ and $y\left(\hat{R}^{(2)}=0\right)$

$$
-\partial^{2} x=\frac{\lambda}{\sqrt{\kappa}} e^{-\frac{2}{\sqrt{\kappa}}(x-y)} \quad, \quad \partial^{2} y=\partial^{2} x
$$


Solving these equations we have to restrict ourselves to solutions that reproduce the $\mathrm{BH}$ solution (10) in the classical limit. Therefore, we are interested in a solution depending on $z^{+} z^{-}$only and find

$$
x=y=-\frac{1}{\sqrt{\kappa}}\left(u-\lambda z^{+} z^{-}\right)
$$

$(u=$ const.). Using the transformation (15) we can express this solution in $\phi$ and $\sigma$. In doing so we have to fix the up to now arbitrary functions $h(\phi)$ and $\bar{h}(\phi)$. Let us start with parameterization suggested by de Alwis: $h=0, \bar{h}=-\frac{1}{2} \kappa e^{2 \phi}$. Motivated is this choice by the fact that for all values of $\phi$ and $\sigma$ the transformation (15) is non singular and secondly that the range of $x$ and $y$ goes from $-\infty$ to $+\infty$ if $\phi$ and $\sigma$ do so. For $x$ and $y$ one gets

$$
\begin{aligned}
& x=\frac{1}{\sqrt{4 \kappa}}\left(-\sqrt{\kappa^{2}+4 e^{-4 \phi}}+\sqrt{\kappa} \operatorname{arcsinh} \frac{\kappa}{2} e^{2 \phi}\right) \\
& y=-\sqrt{\kappa}\left(\sigma-\frac{1}{\kappa} e^{-2 \phi}-\phi\right)
\end{aligned}
$$

In terms of (20) one finds in the weak coupling limit $\left(e^{2 \phi}<<1\right)$ the desired classical solution (10)

$$
e^{-2 \phi}=u-\lambda z^{+} z^{-} \quad, \quad \sigma=\phi .
$$

Since we are in the weak coupling region this solution corresponds to our dual solution (11) which is non-singular for $\eta \simeq 0$. In the strong coupling limit $\left(e^{2 \phi}>>1\right)$ we obtain

$$
\phi=-\frac{1}{\sqrt{\kappa}}\left(u-\lambda z^{+} z^{-}\right) \quad, \quad \sigma=\frac{1}{\kappa} e^{-2 \phi} .
$$

Therefore, after incorporation of quantum corrections $\left.\left(\sim \mathcal{O}(\rceil^{\epsilon \phi}\right)\right)$ the black hole solution gets smooth also in the strong coupling region. Note, that in dilaton gravity a singularity in the metric has to be accompanied by a singularity in the dilaton, i.e. singularities can only appear in the strong or weak coupling region. For the other models the picture is qualitatively the same. In the CGHS model $(h=\bar{h}=0)$ one obtains for (15)

$$
\begin{aligned}
& x=-\frac{1}{\sqrt{\kappa}} e^{-2 \phi} \sqrt{1+\kappa e^{2 \phi}}-\frac{\sqrt{\kappa}}{2} \log \left[\kappa+2 e^{-2 \phi}\left(1+\sqrt{1+\kappa e^{2 \phi}}\right)\right] \\
& y=-\sqrt{\kappa}\left(\sigma-\frac{1}{\kappa} e^{-2 \phi}\right) .
\end{aligned}
$$

and in strong coupling region this model gives

$$
e^{-\phi} \sim u-\lambda z^{+} z^{-} \quad, \quad \sigma=\frac{1}{\kappa}\left(u-\lambda z^{+} z^{-}\right)
$$

For the Strominger model $\left(2 h=\bar{h}=-e^{2 \phi}\right)$ we find $\left(F(\phi)=\sqrt{e^{-4 \phi}-(2-\kappa) e^{-2 \phi}+\frac{2-\kappa}{2}}\right)$

$$
\begin{aligned}
x=-\frac{1}{\sqrt{\kappa}} & {\left[F(\phi)+\frac{\kappa-2}{2} \log \left[F(\phi)+e^{-2 \phi}+\frac{\kappa-2}{2}\right]-\right.} \\
& \left.\quad-\sqrt{\frac{2-\kappa}{2}} \log \left(\sqrt{2(2-\kappa)} F(\phi)+(2-\kappa) e^{2 \phi}-(2-\kappa)\right)\right] \\
y=-\sqrt{\kappa} & \left(\sigma-\frac{1}{\kappa} e^{-2 \phi}-\frac{2}{\kappa} \phi\right) .
\end{aligned}
$$


which gives in the strong coupling region

$$
\phi=-\frac{1}{\sqrt{2(2-\kappa)}}\left(u-\lambda z^{+} z^{-}\right) \quad, \quad \sigma=\frac{1}{\kappa}(\sqrt{2(2-\kappa)}-2) \phi .
$$

And finally in the RST model $\left(h=0, \bar{h}=-\frac{\kappa}{4} e^{2 \phi}\right)$ the general solution is given by

$$
\begin{aligned}
& x=-\frac{1}{\kappa} e^{-2 \phi}+\frac{\kappa}{2} \phi \\
& y=-\sqrt{\kappa}\left(\sigma-\frac{1}{\kappa} e^{-2 \phi}-\frac{1}{2} \phi\right) .
\end{aligned}
$$

In the strong coupling region this model behaves like

$$
\phi=-2 \kappa^{-\frac{3}{2}}\left(u-\lambda z^{+} z^{-}\right) \quad, \quad \sigma=\frac{1-\sqrt{\kappa}}{2} \phi .
$$

Therefore we find that all models have no singularities in the strong coupling region and yield the classical result in the weak coupling region.

One can now ask what is the influence of this quantization procedure for the further evolution of the universe. For the derivation of our results it was crucial that the solution decouples in a $2 \mathrm{~d}$ (dilaton gravity) part and a $3 \mathrm{~d}$ spherical part. This is valid only if one considers the theory, e.g. ,inside the wormhole of fig. 1b. Extending this procedure to the region away from the wormhole seems to be difficult. But nevertheless, quantum correction inside the wormhole can form a dilaton potential which could be a source of an inflationary period in later times. A dilaton potential in our original action (11) or (5) corresponds to an additional tachyon contribution in the $2 \mathrm{~d}$ action which is independent of $\lambda$ (since $\lambda$ was correlated to the constant $\chi$ field in the wormhole, see (8)). The tachyon we have discussed so far is only one possibility. This solution has the advantage that the renormalization group $\beta$ functions vanish thereby yielding a finite $2 \mathrm{~d}$ quantum field theory. The most general tachyon field, however, is a combination of contributions given by (17). A further additive tachyon term is given by (for $\kappa>0$ )

$$
T_{n p}=\mu e^{2 x}
$$

where the function $x$ is given by (15) This term, discussed e.g. in [8] and [10], has in the weak coupling region for all discussed models the typical non-perturbative structure

$$
T_{n p} \sim e^{-\frac{2}{\sqrt{\kappa}} e^{-2 \phi}} \sim e^{-\frac{2}{\sqrt{\kappa} g_{s}^{2}}}
$$

where $g_{s}=e^{\phi}$ is the string coupling constant. Therefore this term vanishes very rapidly in the weak coupling (classical) region and becomes important in the strong coupling region. Furthermore, since $x$ is a function of the dilaton only, this tachyon term represents a candidate for a dilaton potential created by non-perturbative quantum corrections in the strong coupling region. If we insert the $x$ values for the several models (21), (24), (26) and 
(28) we obtain different potentials. But all these potentials have no local or global minima and are probably not good candidates to discuss an inflationary period (see e.g. [11] and refs. therein). It remains an open question whether another choice of the model dependent functions $h$ and $\bar{h}$ could yield a more appropriate potential.

4. Discussion. Starting with a classical solution of the low energy string effective action we investigated the quantization near the cosmological singularity. Via a 5d Kaluza-Klein approach this solution was obtained as a dimensional reduced theory. Near the singularity the $5 \mathrm{~d}$ theory decouples in a $3 \mathrm{~d}$ non singular (spherical) part and a singular $2 \mathrm{~d}$ part. As a first step we have quantized only this singular $2 \mathrm{~d}$ part (s-wave reduction). The results (21) - (29) show that for all models the singularity disappears after the quantization of the theory, i.e. the $2 \mathrm{~d}$ metric part and the dilaton remains finite. An interpretation of this result is that the wormhole becomes traversable via quantum corrections. In addition, we have shown that the incorporation of non perturbative quantum corrections form a dilaton potential. The discussion of the possible structures of the potential created by this procedure remains an interesting task for further investigations.

We used the $5 \mathrm{~d}$ theory to get contact with the known dilaton gravity. But it is also possible to quantize the $4 \mathrm{~d}$ theory (1) directly. Our approximation to quantize only the divergent $2 \mathrm{~d}$ part in 5 dimensions is effectively the same as to quantize the dilaton and moduli matter fields only. Note that the $2 \mathrm{~d}$ metric part has only one degree of freedom. In the conformal gauge (12) this is the Liouville field $\sigma$ but we can also take another gauge, e.g. $d s^{2}=\rho^{2} d w^{2}-d \eta^{2}$ and then $\rho$ is our moduli field (see (6)). Thus, from the $4 d$ point of view we replaced the dilaton and moduli contributions in the Einstein equation by its vacuum expectation value

$$
R_{\mu \nu}^{(E)}-\frac{1}{2} R^{(E)} G_{\mu \nu}^{(E)}=<T_{\mu \nu}^{(\phi, \rho)}>+T_{\mu \nu}^{(H)}
$$

where $G^{(E)}=e^{-2 \phi} G_{\mu \nu}$ is the metric in the Einstein frame. Classically, the $4 \mathrm{~d}$ string metric was smooth but the Einstein metric was singular (caused by the dilaton and moduli). However, after quantization the singularities in the scalar fields disappeared and thereby also the Einstein metric turned out to be non-singular. This implies that similar to the string frame the Einstein metric describes a universe which starts and ends (for $k=1$ ) not with a singularity but with a minimal (nonzero) extension (wormhole). Therefore, in both frames the spatial part of the universe is qualitatively given in figure 1. Of course, the quantization of the scalar fields near the singularity can only be a first step and future investigations have to show whether a complete quantum theory will leave this qualitative feature intact.

\section{Acknowledgments}

We would like to thank S. Förste and J. García-Bellido for useful comments. The work of K.B. is supported by a DAAD grant and of T.T.B. by the Swiss National Science Foundation. The work of both authors was also supported by the US Department of Energy, contract DE-AC03-76SF00515. 


\section{References}

[1] C.G. Callan, S.B. Giddings, J.A. Harvey, A. Strominger: "Evanescent black holes", Phys. Rev. D45 (1992) 1005;

[2] T.T. Burwick, A.H. Chamseddine: "Classical and quantum consideration of 2d gravity", Nucl. Phys. B384 (1992) 411;

[3] A. Strominger: "Faddeev-Popov ghosts and $(1+1)$ dimensional black hole evaporation", Phys. Rev. D46 (1992) 4396;

[4] J.G. Russo, L. Susskind, L. Thorlacius: "End point of Hawking radiation, Phys. Rev. D46 (1992) 3444;

[5] K. Behrndt, S. Förste: "String-Kaluza-Klein cosmology", preprint SLAC-PUB6471, RI-2-94 (hep-th: 9403179); K. Behrndt, S. Förste: "Cosmological string solutions in 4 dimensions from 5d black holes", Phys. Lett. B320 (1994) 253;

[6] G.T. Horowitz, A. Strominger: "Black strings and p-branes", Nucl. Phys. B360 (1991) 197;

[7] E. Witten: "String theory and black holes", Phys. Rev. D44 (1991) 314;

A. Giveon: "Target space duality and stringy black holes", Mod. Phys. Lett. A6 (1991) 2843;

[8] S.P. de Alwis: "Quantum black holes in two dimensions", Phys. Rev. D46 (1992) 5429

[9] A. Bilal, C. Callan: "Liouville model of black hole evaporation", Nucl. Phys. B394 (1993) 73 ;

[10] J.G. Russo, A.A. Tseytlin: "Scalar-tensor quantum gravity in two dimensions", Nucl. Phys. B382 (1992) 259;

[11] R. Brustein: "The role of the superstring dilaton in cosmology and particle physics", preprint CERN-TH.7255/94 (hep-th/9405066). 
This figure "fig1-1.png" is available in "png" format from: http://arxiv.org/ps/hep-th/9407039v1 
This figure "fig1-2.png" is available in "png" format from: http://arxiv.org/ps/hep-th/9407039v1 